Stress separation from photoelastic data by a multigrid method

This article has been downloaded from IOPscience. Please scroll down to see the full text article.

1998 Meas. Sci. Technol. 91204

(http://iopscience.iop.org/0957-0233/9/8/010)

View the table of contents for this issue, or go to the journal homepage for more

Download details:

IP Address: 147.96.14.16

The article was downloaded on 14/05/2013 at 19:04

Please note that terms and conditions apply. 


\section{Stress separation from photoelastic data by a multigrid method}

\section{J A Quiroga and A González-Cano}

Departamento de Óptica, Facultad de Ciencias Físicas, Universidad Complutense, Ciudad Universitaria s/n, 28040 Madrid, Spain

Received 30 January 1998, in final form 14 April 1998, accepted for publication 23 April 1998

Abstract. An application of multigrid techniques for the separation of principal stresses in plane stress systems is presented. By establishing the equilibrium equations and determining the value of shear from photoelastic data a system of partial differential equations is obtained, which can be solved by applying a multigrid method. Multigrid methods are comparable to Fourier methods in efficiency and robustness and can be applied to processing areas of arbitrary shape.

Keywords: photoelasticity, stress measurement, multigrid techniques

\section{The problem of stress separation}

The determination of the values of the principal stresses at every point in a sample from any kind of direct experimental measurements is called stress separation. In photoelastic experiments it is not possible to obtain the values of the principal stresses in every point of the sample directly. What is obtained is the difference of the principal stresses (associated with isochromatics) and their orientations (associated with isoclinics) [1,2]. Therefore, some information must be added to photoelastic data to separate stresses. For instance, we can experimentally determine the value of the sum of principal stresses by interferometric techniques [2,3]. Another possibility is the so-called oblique incidence method. For this technique different transmission directions are used giving different retardation measurements, from which it is possible to compute the principal stresses [1]. The method that we have chosen is the resolution of the equilibrium equations, which are relationships between the spatial variations of the Cartesian components of the stresses and the value of the shear stress at every point. The connecting point between photoelastic data and the equilibrium equations is the shear stress, because it can be directly measured by photoelasticity [1].

In this way, if $\sigma_{x}$ and $\sigma_{y}$ are the components of the stress at a given point in a fixed Cartesian system of reference and $\sigma_{x y}$ is the shear stress, the equilibrium equations, in the absence of body forces, are given by

$$
\begin{aligned}
& \frac{\partial \sigma_{x}}{\partial x}+\frac{\partial \sigma_{x y}}{\partial y}=0 \\
& \frac{\partial \sigma_{y}}{\partial y}+\frac{\partial \sigma_{x y}}{\partial x}=0
\end{aligned}
$$

Now, if $\sigma_{1}$ and $\sigma_{2}$ are the principal stresses at that point and $\alpha$ is the isoclinic angle, which corresponds to the local orientation of the principal stresses, the following relationships hold:

$$
\begin{gathered}
\sigma_{x}-\sigma_{y}=\left(\sigma_{1}-\sigma_{2}\right) \cos (2 \alpha) \\
\sigma_{x y}=\frac{1}{2}\left(\sigma_{1}-\sigma_{2}\right) \sin (2 \alpha) .
\end{gathered}
$$

The difference of the principal stresses can be related to the isochromatic phase $\delta$, which is obtained in photoelastic experiments, by

$$
\sigma_{1}-\sigma_{2}=\frac{\lambda}{2 \pi d C} \delta
$$

where $C$ is the photoelastic constant of the material, $d$ the thickness of the sample and $\lambda$ the wavelength.

If we now define

$$
K=\frac{\lambda}{2 \pi d C}
$$

and two new variables

$$
\begin{aligned}
& \Phi=K \delta \cos (2 \alpha) \\
& \Psi=K \delta \sin (2 \alpha)
\end{aligned}
$$

we have

$$
\begin{gathered}
\frac{\partial \sigma}{\partial x}=-\frac{\partial \Phi}{\partial x}-\frac{\partial \Psi}{\partial y} \\
\frac{\partial \sigma}{\partial y}=\frac{\partial \Phi}{\partial y}-\frac{\partial \Psi}{\partial x}
\end{gathered}
$$

where $\sigma$ is the sum of the principal stresses, $\sigma=\sigma_{1}+\sigma_{2}=$ $\sigma_{x}+\sigma_{y}$. It is interesting to note that this sum satisfies the Laplace equation,

$$
\nabla^{2} \sigma=0
$$


where $\nabla$ is the Nabla operator. It is known that in this case $\sigma$ can be determined in any region if we know its values on the boundary of that region [4].

From (6) we can obtain the sum of principal stresses except for a constant of integration that must be determined by some a priori knowledge of the state of stress (boundary conditions) or from another independent measurement. Since we have by (3) the difference of the principal stresses obtained from the isochromatics and from (6) the sum, we can determine $\sigma_{1}$ and $\sigma_{2}$ (or $\sigma_{x}$ and $\sigma_{y}$ ).

We must then solve (6), a system of partial differential equations in which the right-hand side is a set of experimental values, which are of course discrete. We see that the problem of stress separation is reduced to a problem of integration of pairs of difference equations, which correspond to the discrete version of equation (6). Many numerical methods can be applied to achieve this goal [4-9]. When dealing with stress separation these methods must be capable of working with arbitrarily shaped processing areas and of overcoming the problems associated with the noise in the data. Noise problems are especially significant, since we measure $\Phi$ and $\Psi$ and then we calculate their partial derivatives and mix them, so the noise of the experimental data is amplified.

Two different approaches to the stress separation by the integration of equations (6) should be mentioned. First, a line integration is presented in the work of Haake et al [10]. The main problem of this technique is that the variance of the reconstruction error is proportional to the number of points processed [11]. Also, the starting points for the line integration are usually points on the border of the processing area, which are frequently the most problematic points. This results in a noisy (line by line) distribution of stresses in the whole body of the sample. Finally, this is a local method that does not take into account the global structure of the derivatives, resulting in the possibility that some local problems can be extended to the whole processing area.

On the other hand, Mahfuz et al have applied a successive over-relaxation (SOR) technique [12]. In this case, the main problem is the slow convergence of the algorithm-it goes as $O\left(N^{3}\right)$, where $N$ is the total number of processed points -, which is very inadequate in practical applications, in which the typical size of the images to be processed is $512 \times 512$ pixels.

\section{Application of multigrid methods to stress separation}

We present here a new method of stress separation that is based on multigrid techniques [13]. These are methods of solving partial differential equation on large grids, based on the idea of applying Gauss-Seidel relaxation schemes on coarser smaller grids.

From the experimental photoelastic data $\alpha$ and $\delta$ we can compute $\Phi$ and $\Psi$ at every point, thus obtaining discrete sets of values $\Phi_{i j}$ and $\Psi_{i j}$, where $i$ and $j$ are the indices that correspond to the columns and the rows of the image, respectively. If we calculate the first differences of these points and combine them in the way that appears in

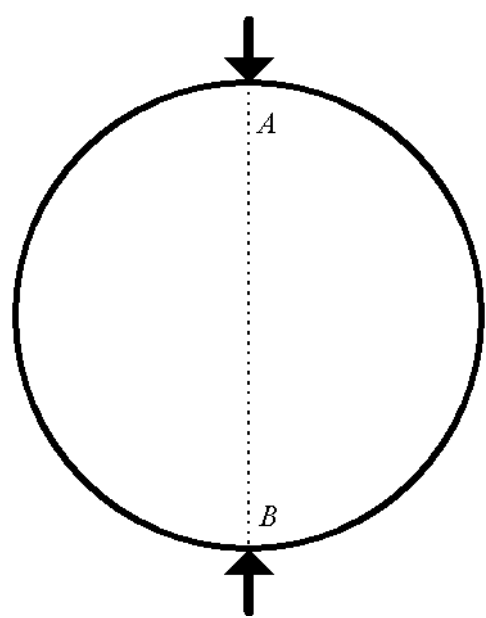

Figure 1. A schematic diagram of the diametrically loaded disc used to test the algorithms. The arrows show the points where the loads have been applied.

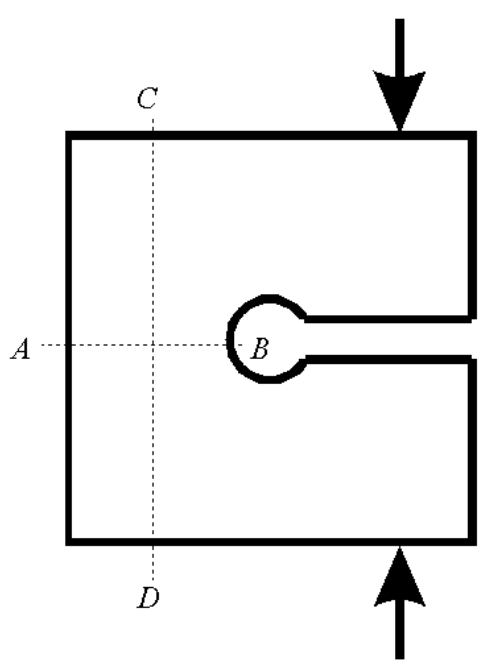

Figure 2. A schematic diagram of the second sample used to test the algorithms. The sample consists of a plate with a hole and a cut joining the hole with the border. The arrows show the points where the loads have been applied. The line $C D$ is at a distance from the border of the hole equal to one diameter of the hole.

equations (6), we will obtain a discrete estimation of the partial derivatives of the sum of the principal stresses, $\sigma$, with respect to $x$ and $y$, that we will denote by $\Delta_{i j}^{x}$ and $\Delta_{i j}^{y}$ :

$$
\begin{aligned}
\Delta_{i j}^{x} & =-D_{x} \Phi_{i j}-D_{y} \Psi_{i j} \\
\Delta_{i j}^{y} & =-D_{y} \Phi_{i j}-D_{x} \Psi_{i j}
\end{aligned}
$$

where we are assuming square pixels with $\Delta x=\Delta y$ and $D_{x}$ and $D_{y}$ are the discrete first-difference operators defined as

$$
\begin{aligned}
& D_{x} f_{i j}=f_{i j}-f_{i-1, j} \\
& D_{y} f_{i j}=f_{i j}-f_{i, j-1} .
\end{aligned}
$$

From the estimations $\Delta_{i j}^{x}$ and $\Delta_{i j}^{y}$ we must obtain a set of values $\sigma_{i j}$ whose partial first differences fit best to $\Delta_{i j}^{x}$ and $\Delta_{i j}^{y}$. We must take into account that the values of 


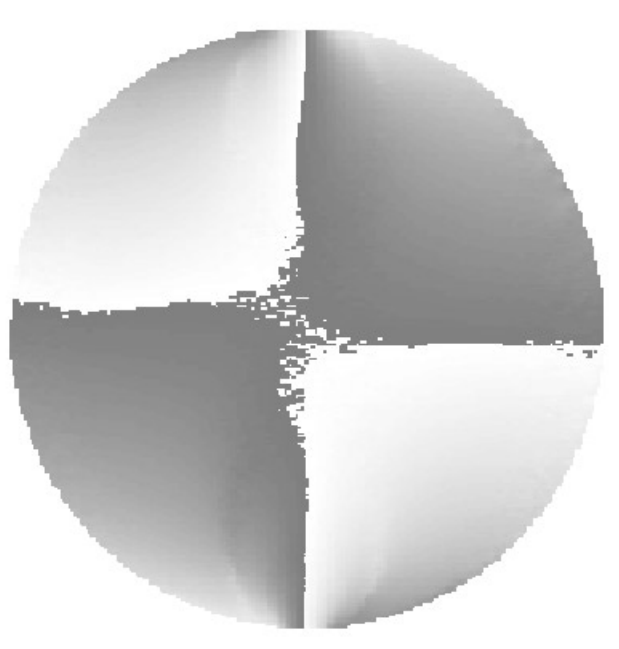

(a)
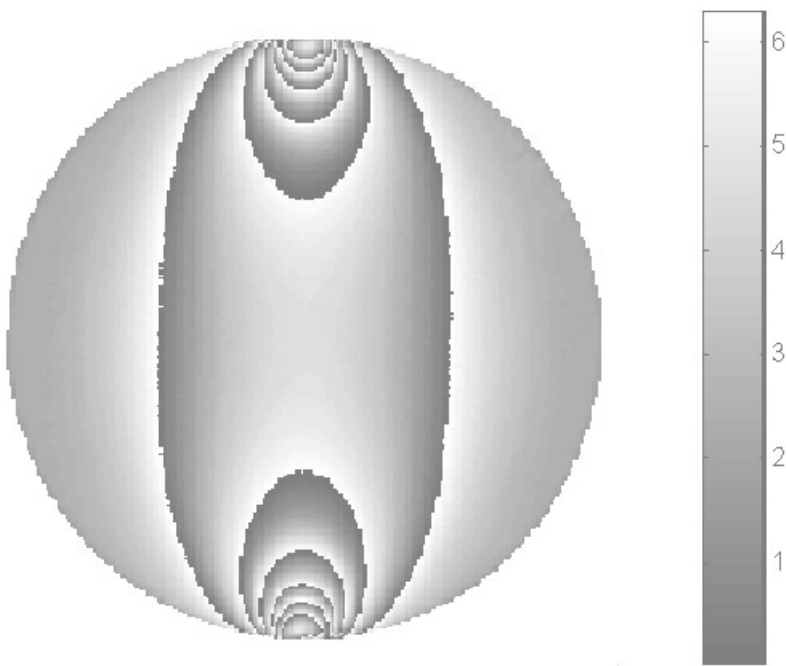

(b)

Figure 3. Distributions of isoclinics, $4 \alpha$ (modulo $2 \pi$ ) (a) and isochromatics, $\delta$ (modulo $2 \pi$ ) (b) for the disc of figure 1 . The scale is in radians.

$\Delta_{i j}^{x}$ and $\Delta_{i j}^{y}$ come from experimental measures and are in principle noisy. This will mean that, for some points of the grid (that is, for some values of $i, j$ ) the values of $\Delta_{i j}^{x}$ and $\Delta_{i j}^{y}$ will not be reliable. For this reason, we must choose a criterion to obtain the best estimation of $\sigma_{i j}$ from our set of experimental values of differences. We will impose a least-squares criterion [14]: the gradient of the field $\sigma_{i j}$ must minimize the energy functional

$$
U=\sum_{i, j}\left(D_{x} \sigma_{i j}-\Delta_{i j}^{x}\right)^{2}+\sum_{i, j}\left(D_{y} \sigma_{i j}-\Delta_{i j}^{y}\right)^{2} .
$$

It can be shown [15] that the function $\sigma_{i j}$ that minimizes $U$ is the solution of a Poisson equation given by

$$
\nabla^{2}\left(\sigma_{i j}\right)=\rho_{i j}
$$

where $\nabla^{2}$ denotes the Laplacian operator, that in discretized form is given by

$$
\left(\sigma_{i+1, j}-2 \sigma_{i j}+\sigma_{i-1, j}\right)+\left(\sigma_{i, j+1}-2 \sigma_{i j}+\sigma_{i, j-1}\right)=\rho_{i j}
$$

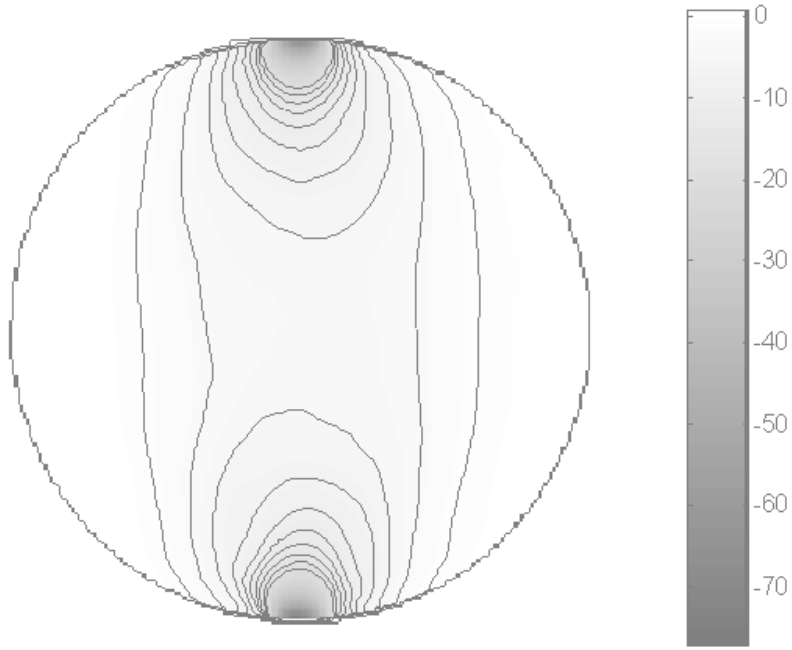

(a)
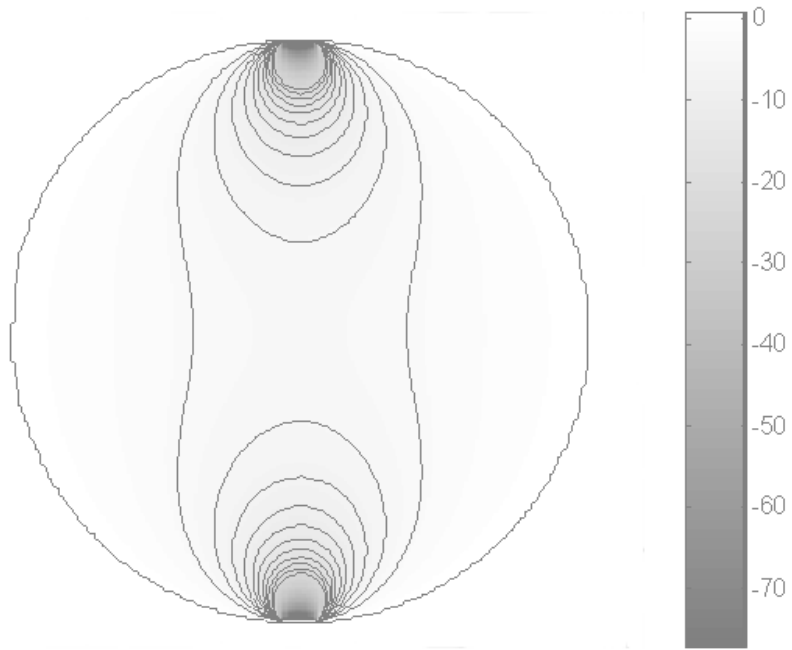

(b)

Figure 4. Distributions of the sum of principal stresses, $\sigma$, for the disc of figure 1, obtained with our multigrid method (a) and theoretically (b). Contour lines are included for the sake of clarity. In this and all the following figures the units for the stresses are arbitrary.

and

$$
\rho_{i j}=\Delta_{i+1, j}^{x}-\Delta_{i j}^{x}+\Delta_{i, j+1}^{y}-\Delta_{i j}^{y} .
$$

The classical method of solving equation (11) is the so-called Gauss-Seidel relaxation method. In this method the array $\sigma_{i j}$ is initialized to zero and then the following updates are performed in an iterative fashion:

$$
\sigma_{i j}=\frac{1}{4}\left[\left(\sigma_{i+1, j}+\sigma_{i-1, j}+\sigma_{i, j+1}+\sigma_{i, j-1}\right)-\rho_{i j}\right] .
$$

The problem is that the Gauss-Seidel relaxation converges too slowly, even if optimizations such as the so-called redblack method are used [4].

Equation (14) is usually solved by Fourier methods when all the points of the rectangular grid are valid. However, in realistic cases not all points are valid. To take into account this fact we can introduce some weights that corresponds to the degree of reliability of each point, $0 \leq w_{i j} \leq 1$. We can adopt, for instance, a binary mask in 


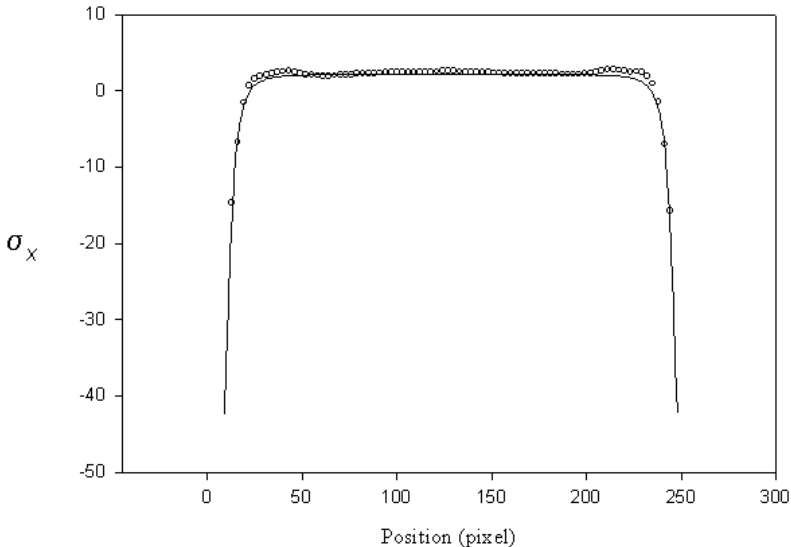

(a)

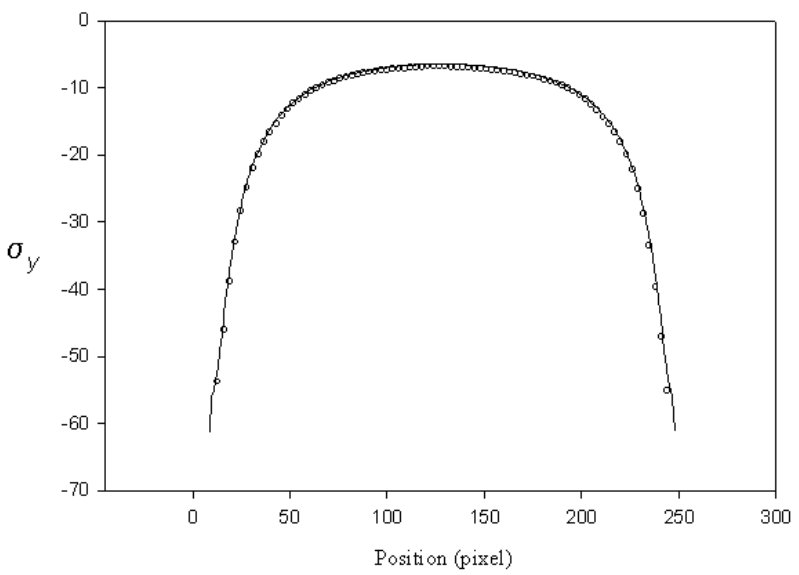

(b)

Figure 5. Profiles of the principal stresses $\sigma_{x}(\mathrm{a})$ and $\sigma_{y}(\mathrm{~b})$ for the line $A B$ of the disc of figure 1 . The theoretical result is represented as a full line. The circles represent the points obtained with our algorithm.

which the invalid pixels are given a weight 0 and a weight 1 is given to the valid points. If we do this, we obtain a different Poisson equation and a corresponding GaussSeidel relaxation equation [14],

$$
\begin{aligned}
\sigma_{i j}= & \frac{1}{v_{i j}}\left[\left(w_{i+1, j}^{x} \sigma_{i+1, j}+w_{i j}^{x} \sigma_{i-1, j}+w_{i, j+1}^{y} \sigma_{1, j+1}\right.\right. \\
& \left.\left.+w_{i j}^{y} \sigma_{i, j-1}\right)-\hat{\rho}_{i j}\right]
\end{aligned}
$$

where

$$
\begin{gathered}
w_{i j}^{x}=\min \left(w_{i j}^{2}, w_{i-1, j}^{2}\right) \quad w_{i j}^{y}=\min \left(w_{i j}^{2}, w_{i, j-1}^{2}\right) \\
v_{i j}=w_{i+1, j}^{x}+w_{i j}^{x}+w_{i, j+1}^{y}+w_{i j}^{y} \\
\hat{\rho}_{i j}=w_{i+1, j}^{x} \Delta_{i+1, j}^{x}-w_{i j}^{x} \Delta_{i j}^{x}+w_{i, j+1}^{y} \Delta_{i, j+1}^{y}-w_{i j}^{y} \Delta_{i j}^{y} .
\end{gathered}
$$

Equation (15) cannot be solved in an easy way using Fourier methods; it must be solved iteratively.

Gauss-Seidel relaxation provides the high-frequency details of the function very fast, but it propagates this information very slowly, since in each step of the iteration each point receives information only of its immediate neighbours, so that low-frequency features of the function are difficult to obtain. For this reason, multigrid methods
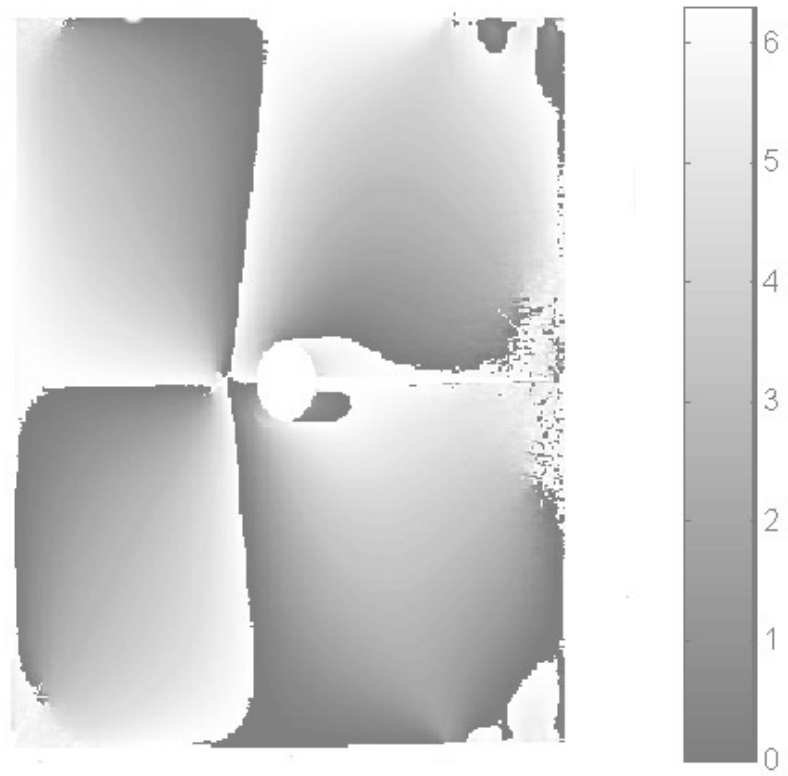

(a)
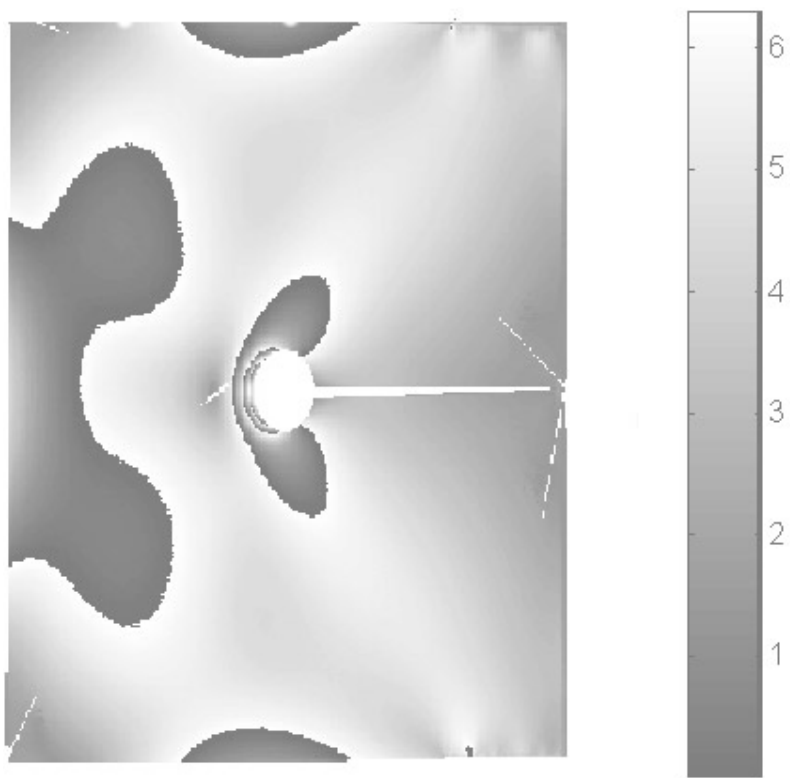

(b)

Figure 6. Distributions of isoclinics, $4 \alpha$ (modulo $2 \pi$ ) (a) and isochromatics, $\delta$ (modulo $2 \pi$ ) (b) for the plate of figure 2 . The scale is in radians.

are especially well suited to improve the efficiency of the Gauss-Seidel relaxation scheme.

The key idea of the multigrid approach is to transform the low-frequency components of the solution obtained by a Gauss-Seidel relaxation into high-frequency components of a coarser grid. With a coarser grid, Gauss-Seidel relaxation works very well and we need only translate the results obtained to the finer grids. A set of grids, each with a double spacing between points, can be used and the information must be transferred first from finer to coarser and then from coarser to finer grids. This is accomplished by the action of two operators, prolongation and restriction. The specific details of the multigrid algorithm and the forms 

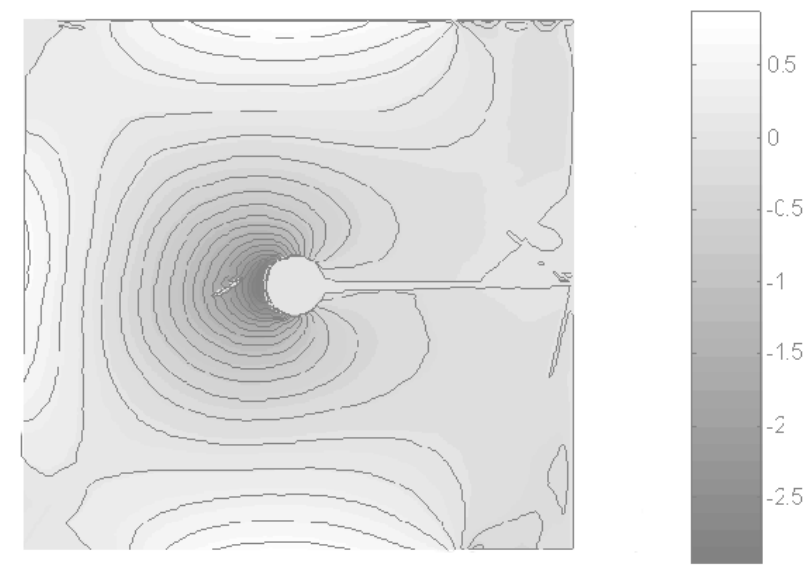

(a)
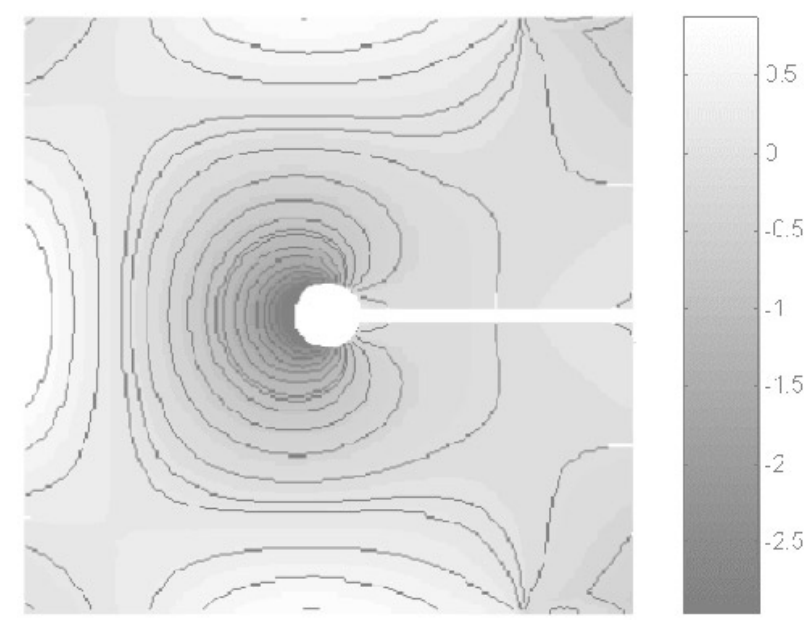

(b)

Figure 7. Distributions of the sum of principal stresses, $\sigma$, for the plate of figure 2, obtained with our multigrid method (a) and with a finite-element technique (b). Contour lines are included for the sake of clarity.

of these operators, as well as a pseudo-code listing, can be found in [14].

Multigrid methods are comparable in speed to Fourier methods and can be used with arbitrarily shaped processing areas. Also, these algorithms are very easily implementable. Therefore these methods are a good choice to perform the integration of pairs of differences with realistic image sizes (up to $512 \times 512$ pixels) and noise levels. The results obtained are good, as the examples of the next section show.

\section{Experimental results}

To illustrate the performance of our method we have chosen two examples. The first one is quite academic: a diametrically compressed disc (figure 1). The image size was $256 \times 256$ points. A more realistic sample is used for the second example: a plate with a hole and a cut joining the hole with the border (image size $512 \times 512$ pixels). A compressive force is applied as indicated in figure 2 .

In figure 3 the distributions of isoclinics (a) and isochromatics (b) for the disc are shown. These parameters

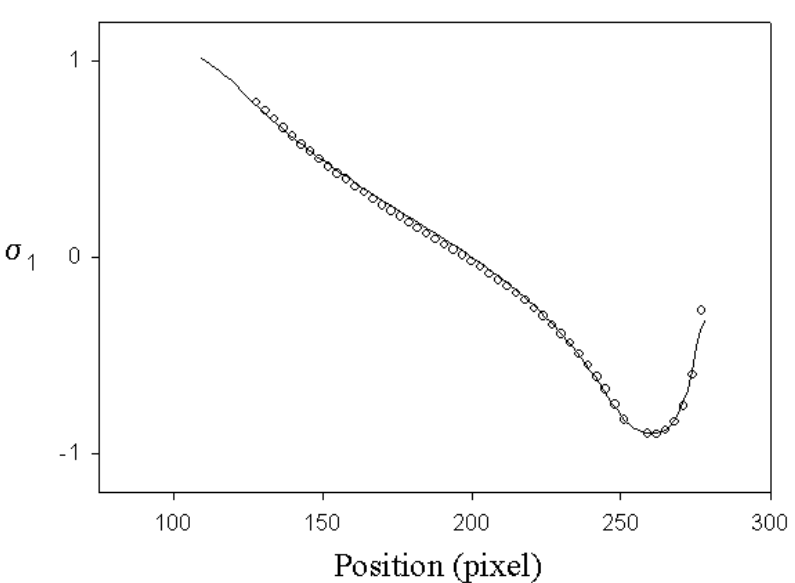

(a)

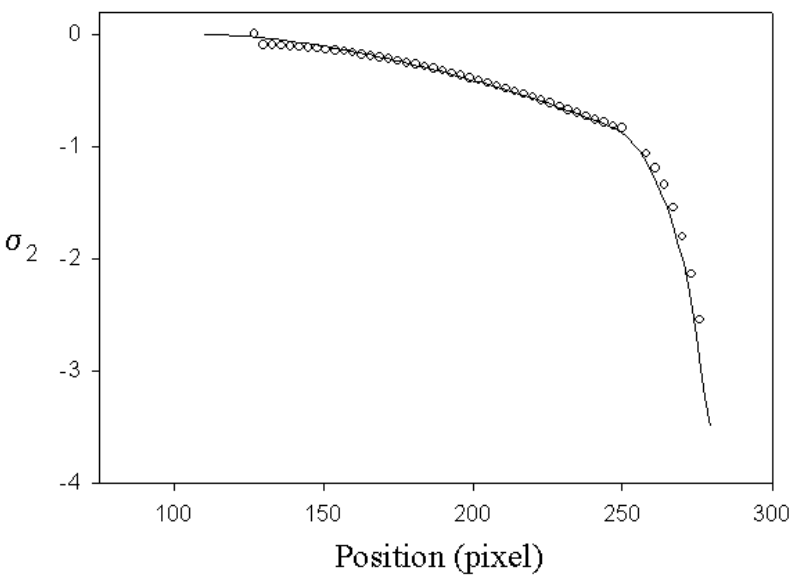

(b)

Figure 8. Profiles of the principal stresses $\sigma_{1}$ (a) and $\sigma_{2}$ (b) for the line $A B$ in the plate of figure 2. The circles represent the points obtained with our algorithm and the results obtained with the finite-element technique are represented as a full line.

can be determined using various algorithms [16-18]. We have used the one depicted in [18]. This is a phase-shiftbased algorithm in which the retardation and the angle of isoclinics are computed by an arctangent computation, in this way we measure modulo $2 \pi$ the retardation $\delta$ and four times the isoclinic angle $4 \alpha$ as figures 3 and 6 show. The fundamental difficulty associated with the accurate location of isoclinics is overcome by these phaseshift-based algorithms because the arctangent calculation is performed from the values of intensity at every point of the area of interest, so we do not need to locate the isoclinic fringes explicitly.

In figure 4(a) we show the distribution of the sum of principal stresses, $\sigma=\sigma_{1}+\sigma_{2}$, obtained with the multigrid method. For purposes of comparison, we show in figure 4(b) that distribution computed theoretically [1]. In figure 5 the theoretical and experimental profiles of the distributions of principal stresses $\sigma_{1}$ (a) and $\sigma_{2}$ (b) along the line $A B$ of figure 1 are shown. The reconstruction error may be computed by evaluating

$$
E=\frac{\sum_{i, j}\left(D_{x} \sigma_{i j}-\Delta_{i j}^{x}\right)^{2}+\sum_{i, j}\left(D_{y} \sigma_{i j}-\Delta_{i j}^{y}\right)^{2}}{\sum_{i, j}\left[\left(\Delta_{i j}^{x}\right)^{2}+\left(\Delta_{i j}^{y}\right)^{2}\right]}
$$




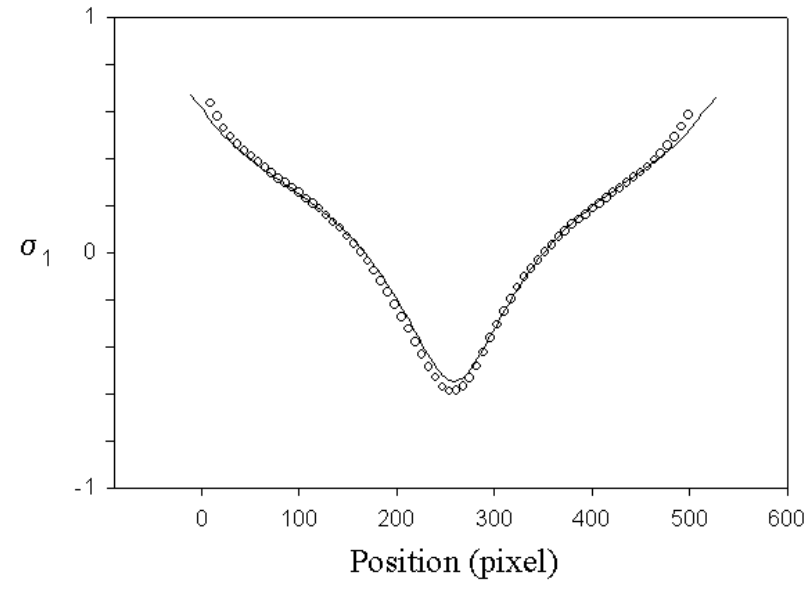

(a)

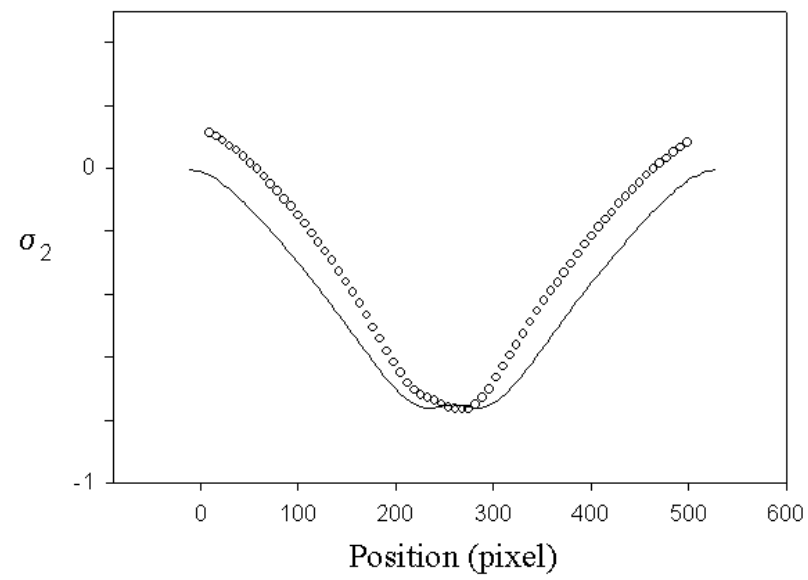

(b)

Figure 9. Profiles of the principal stresses $\sigma_{1}$ (a) and $\sigma_{2}$ (b) for the line $C D$ in the plate of figure 2 . The circles represent the points obtained with our algorithm and the results obtained with the finite-element technique are represented as a full line.

that measures the total normalized least-squares error. Also, we can compute the corresponding errors in $x$ and $y$ :

$$
\begin{aligned}
& E_{x}=\frac{\sum_{i, j}\left(D_{x} \sigma_{i j}-\Delta_{i j}^{x}\right)^{2}}{\sum_{i, j}\left(\Delta_{i j}^{x}\right)^{2}} \\
& E_{y}=\frac{\sum_{i, j}\left(D_{y} \sigma_{i j}-\Delta_{i j}^{y}\right)^{2}}{\sum_{i, j}\left(\Delta_{i j}^{y}\right)^{2}} .
\end{aligned}
$$

In the case of the disc the values for the errors were $E=0.0636, E_{x}=0.0661$ and $E_{y}=0.0607$.

Results of the same type are obtained for the other sample. In figure 6 we show the distributions of isoclinics (a) and isochromatics (b). In figure 7(a) the distribution of $\sigma$ is represented. In this case we do not have the theoretical distribution and we have tested the validity of our algorithm with a finite-element technique, whose result is shown in figure 7(b). In figures 8(a) and (b) the profiles of $\sigma_{x}$ and $\sigma_{y}$ are shown for both results for the line $A B$ of figure 2. The profiles of $\sigma_{x}$ and $\sigma_{y}$ for the line $C D$ are shown in figures 9 (a) and (b). The reconstruction errors were $E=0.1166, E_{x}=0.1194$ and $E_{y}=0.1142$.
As these results show, the performance of the multigrid method for the stress separation is good. For both samples, the number of multigrid cycles required has been three, with a total number of Gauss-Seidel relaxations at each grid level of four. The total computing time was $13.36 \mathrm{~s}$ for the disc and $60 \mathrm{~s}$ for the plate. We can see that this method is useful for this type of problem.

The multigrid algorithm has been implemented under the MATLAB environment as MEX files. The programs have been run on a Pentium PC at $120 \mathrm{MHz}$.

We can see that multigrid methods are well suited to the process of stress separation from photoelastic data. The use of this method, together with the phasemeasuring algorithms for determination of isoclinics and isochromatics, permits the complete evaluation of the stress state of a plane sample in a few minutes.

\section{Acknowledgments}

We thank Professor Eusebio Bernabeu, director of the Optics Department of the Universidad Complutense for his help and continuous support. Also, we thank Hans Steinbichler, of Steinbichler Optotechnik GmbH, for his technical assistance. This work has been partially supported by project MAT 95-0767 of the CICYT (Comision Interministerial de Ciencia y Tecnología).

\section{References}

[1] Frocht M M 1941 and 1948 (volumes 1 and 2) Photoelasticity (New York: Wiley)

[2] Theocaris P S and Gdoutos E E 1979 Matrix Methods in Photoelasticity (Berlin: Springer)

[3] Brown G M and Sullivan J L 1990 The computer aided holophotoelastic method: theory and experiment Proc. SEM Conf. on Hologram Interferometry and Speckle Metrology (Baltimore, MD, 5-8 November 1990) (Bethel, CN: Society for Experimental Mechanics) pp 102-9

[4] Press W H, Teukolsky S A, Vetterling W T and Flannery B P 1992 Numerical Recipes in C. The Art of Scientific Computing 2nd edn (Cambridge: Cambridge University Press)

[5] Servin M, Malacara D and Marroquin J L 1996 Wave-front recovery from two orthogonal sheared interferograms Appl. Opt. 35 4343-8

[6] van Brug H 1997 Zernike polynomials as a basis for wave-front fitting in lateral shearing interferometry Appl. Opt. 36 2788-90

[7] Grèdiac M 1997 Method for surface reconstruction from slope or curvature measurements of rectangular areas Appl. Opt. 36 4823-9

[8] Loheide S 1997 General evaluation technique for shearing experiments Fringe '97. Automatic Processing of Fringe Patterns ed W Jüptner and W Osten (Berlin: Akademie) pp 92-4

[9] Rivera M, Marroquin J L, Servin M and Rodriguez-Vera R 1997 Fast algorithm for integrating inconsistent gradient fields Appl. Opt. 36 8381-90

[10] Haake S J, Patterson E A and Wang Z F 1996 2D and 3D separation of stresses using automated photoelasticity Exp. Mech. 36 269-76

[11] Freischlad K 1992 Wavefront integration from difference data Proc. SPIE 1755 212-18

[12] Mahfuz H, Wong T L and Case R O 1990 Separation of principal stresses by SOR technique over arbitrary boundaries Exp. Mech. 30 319-27 
[13] Briggs W L 1987 A Multigrid Tutorial (Philadelphia, PA: SIAM)

[14] Pritt M D 1996 Phase unwrapping by means of multigrid techniques for interferometric SAR IEEE Trans. Geosci. Remote Sensing 34 728-38

[15] Takajo H and Takahashi T 1988 Least-squares phase estimation from the phase difference J. Opt. Soc. Am. A 5 416-25
[16] Asundi A 1993 Phase shifting in photoelasticity Exp. Tech. 17 19-23

[17] Nurse A D 1997 Full-field automated photoelasticity by use of a three-wavelength approach to phase stepping Appl. Opt. 36 5781-6

[18] Quiroga J A and González-Cano A 1997 Phase measuring algorithm for extraction of isochromatics of photoelastic fringe patterns Appl. Opt. 36 8397-402 